\title{
High-Frequency Noise Sources in Quantum Wells
}

\author{
L. Ardaravičius*, J. Liberis and A. Matulionis \\ Semiconductor Physics Institute, 11 A. Goštauto, Vilnius 2600, Lithuania
}

\begin{abstract}
Hot-electron noise is investigated for InGaAs and InAs quantum wells containing a two-dimensional electron gas channel in a pulsed electric field applied parallel to the interfaces. Noise sources resulting from hot-electron "thermal" motion, electron temperature fluctuations, and real-space transfer are observed. The experimental results on hot-electron "thermal" noise are used to estimate energy relaxation time in the field range where other sources do not play any important role. Measurements of noise anisotropy in the plane of electron confinement are used to discuss real-space-transfer noise. High-frequency noise technique is used to study hot-electron trapping, and trap location in InAlAs/InGaAs/InAlAs heterostructure channels is determined.
\end{abstract}

PACS numbers: $72.20 . \mathrm{Ht}, 72.70 .+\mathrm{m}, 73.40 . \mathrm{Kp}$

\section{Introduction}

Compound semiconductor heterostructures containing a two-dimensional electron gas (2DEG) are of interest for fundamental research and for applications in ultra high-speed opto- and microelectronics [1]. Electron heating by high electric fields applied in the plane of electron confinement introduces excess noise such as hot-electron "thermal" noise [2], real-space-transfer noise [2, 3], trap-related noise [4] and others. In this paper, results for InGaAs and InAs quantum well channels are presented and discussed in terms of fast kinetic processes.

\section{2DEG structures and experimental}

Two types of MBE-grown heterostructures, namely, InAlAs/ $/ n_{x} \mathrm{Ga}_{1-x} \mathrm{As} /$ InAlAs and GaSb/AlSb/InAs/AlSb/GaSb, were studied [2, 3, 5] (for 2DEG den-

*corresponding author; e-mail: linas@uj.pfi.lt 
sity $n$ and mobility $\mu$ see Tables I and II). In the $\operatorname{In}_{x} \mathrm{Ga}_{1-x}$ As-containing structures, the mobile electrons were supplied to the quantum well by the donor planes located in the InAlAs barrier layer(s), and the well depth was changed varying doping and composition $x$. The lattice matched heterostructures (13Q) and strained heterostructures (14Q and 15Q) were subcritically doped, so that at equilibrium all mobile electrons resided in the InGaAs quantum-well channel (the estimated critical donor density for the appearance of two-channel conduction is $\approx 5 \times 10^{12} \mathrm{~cm}^{-2}$, Fig. 1). The lattice-matched InGaAs structure H090 was supercritically doped, that is, the high-mobility $\left(\mu_{1}\right)$ InGaAs channel shared the mobile electrons with

\section{TABLE I}

Electron mobility and density at $80 \mathrm{~K}$.

\begin{tabular}{l|c|c|c|c|c}
\hline \hline \multicolumn{1}{c|}{ Structure } & Ref. & $\begin{array}{c}\mu(80 \mathrm{~K}) \\
{\left[\mathrm{cm}^{2} /(\mathrm{V} \cdot \mathrm{s})\right]}\end{array}$ & $\begin{array}{c}n(80 \mathrm{~K}) \\
10^{12}\left[\mathrm{~cm}^{-2}\right]\end{array}$ & $\begin{array}{c}\mu(300 \mathrm{~K}) \\
{\left[\mathrm{cm}^{2} /(\mathrm{V} \cdot \mathrm{s})\right]}\end{array}$ & $\begin{array}{c}n(300 \mathrm{~K}) \\
10^{12}\left[\mathrm{~cm}^{-2}\right]\end{array}$ \\
\hline $13 \mathrm{Q}$ & {$[5]$} & 37000 & 2.4 & 10600 & 2.4 \\
$14 \mathrm{Q}$ & {$[5]$} & 37000 & 2.4 & 11200 & 2.3 \\
$15 \mathrm{Q}$ & {$[5]$} & 11900 & 4.3 & 7260 & 4.1 \\
InAs/AlSb/GaSb & {$[3]$} & 7260 & 0.9 & 7260 & 1.5
\end{tabular}

TABLE II

Electron mobility and density for supercritically doped InGaAs heterostructure at $300 \mathrm{~K}$.

\begin{tabular}{l|c|c|c|c|c|c|c}
\hline \hline Structure & Ref. & $\begin{array}{c}\mu \\
{\left[\mathrm{cm}^{2} /(\mathrm{V} \cdot \mathrm{s})\right]}\end{array}$ & $\begin{array}{c}\mu_{1} \\
{\left[\mathrm{~cm}^{2} /(\mathrm{V} \cdot \mathrm{s})\right]}\end{array}$ & $\begin{array}{c}\mu_{2} \\
{\left[\mathrm{~cm}^{2} /(\mathrm{V} \cdot \mathrm{s})\right]}\end{array}$ & $\begin{array}{c}n \\
10^{12} \\
{\left[\mathrm{~cm}^{-2}\right]}\end{array}$ & $\begin{array}{c}n_{1} \\
10^{12} \\
{\left[\mathrm{~cm}^{-2}\right]}\end{array}$ & $\begin{array}{c}n_{1} \\
10^{12} \\
{\left[\mathrm{~cm}^{-2}\right]}\end{array}$ \\
\hline H090 & {$[2]$} & 4500 & 1200 & 5000 & 33 & 8.5 & 24.5 \\
\hline
\end{tabular}

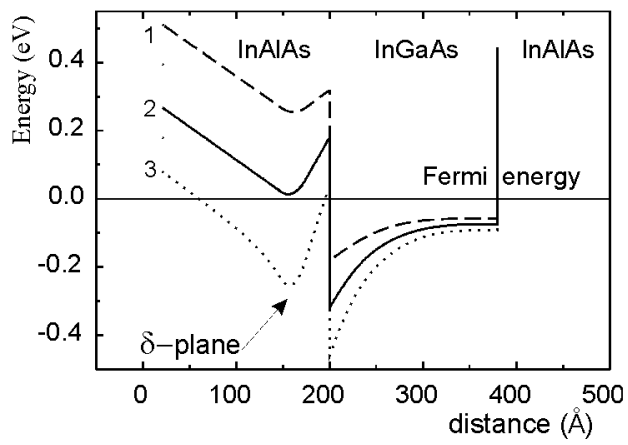

Fig. 1. A schematic view of the band diagram at different donor density for InAlAs/InGaAs/InAlAs [5] at $80 \mathrm{~K}$; (1) $3.1 \times 10^{12} \mathrm{~cm}^{-2}$; (2) $5 \times 10^{12} \mathrm{~cm}^{-2}$; (3) $11.9 \times 10^{12} \mathrm{~cm}^{-2}$. 
the low-mobility InAlAs channel $\left(\mu_{2}\right)$ (Fig. 1, Table II, where $\mu$ is the effective mobility).

The second type of the investigated heterostructures were nominally undoped InAs/AlSb/GaSb having quantum wells for a 2DEG in InAs and for a two-dimensional hole gas (2DHG) in GaSb. In this system, the hole mobility and density appear to be lower than the electron mobility and density [6], and the hole contribution can be neglected in transport and noise experiments.

High frequency spectral noise power $P_{\mathrm{n}}$ was measured in the frequency range from $220 \mathrm{MHz}$ to $10 \mathrm{GHz}$ using gated modulated radiometer technique [1]. Under the matched conditions, the evaluation of the equivalent hot-electron noise temperature $T_{\mathrm{n}}$ was obtained from the relationship: $T_{\mathrm{n}}=P_{\mathrm{n}} / k_{\mathrm{B}} \Delta f$, where $k_{\mathrm{B}}$ is the Boltzmann constant, and $\Delta f$ is the frequency band width. The pulsed electric field was applied parallel to the heterointerfaces. The pulse duration $(2 \mu \mathrm{s})$ and the repetition rate of the pulses $(75 \mathrm{~Hz})$ were chosen in order to avoid lattice heating.

\section{Hot-electron noise and fast/ultrafast processes in a 2DEG}

\subsection{Hot-electron "thermal" noise and real-space transfer}

In the presence of a sufficiently strong electric field (hot-electron case), the Ohm law no longer holds, and the Nyquist theorem fails. The electron chaotic motion causes velocity fluctuations in all directions in the plane of electron confinement. The associated noise was resolved by measuring microwave noise in the direction perpendicular and parallel to the applied electric field. The data for the heterostructure H090 are presented in Fig. 2.

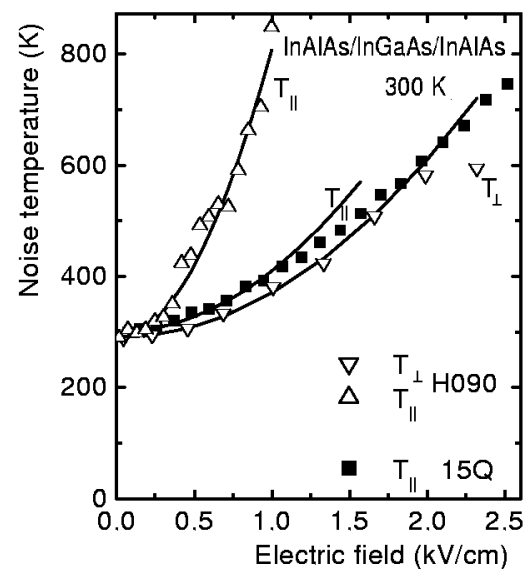

Fig. 2. The transverse $T_{\perp}$ and longitudinal $T_{\|}$noise temperature dependence on the applied electric field at $300 \mathrm{~K}$ for structures H090 and 15Q [2, 5]. The solid line curves represent parabolic approximations. 
As mentioned, two-channel conduction takes place in the H090 heterostructure. Channel occupancy fluctuations due to real-space transfer contribute to the excess noise in the current direction [5], and the longitudinal noise temperature $T_{\mathrm{n} \|}$ exceeds the transverse one [2] (Fig. 2). Noise anisotropy measurements yield contributions due to hot-electron "thermal" noise and due to real-space transfer. The longitudinal noise temperature $T_{\mathrm{n} \|}$ for the subcritically doped structure $15 \mathrm{Q}$ is about the same as $T_{\mathrm{n} \perp}$ for the supercritically doped structure H090 (Fig. 2). This gives a strong evidence that the real-space-transfer noise is weak in $15 \mathrm{Q}$ in the considered range of the electric fields.

\subsection{Hot-electron "cooling"}

Hot-electron "cooling" means that the noise temperature of the electrons in the applied electric field is lower than the lattice temperature [7]. This phenomenon was observed in In As/AlSb/GaSb quantum-well heterostructure by measuring $T_{\mathrm{n}}$ at $80 \mathrm{~K}$ (Fig. 3). Indeed, in the range of the electric fields not exceeding $200 \mathrm{~V} / \mathrm{cm}$, the hot-electron longitudinal noise temperature $T_{\mathrm{n} \|}$ is lower than the lattice temperature. At the same time, the differential resistance undergoes a considerable change, which means that the electric field is strong enough for the non-ohmic behavior to appear.

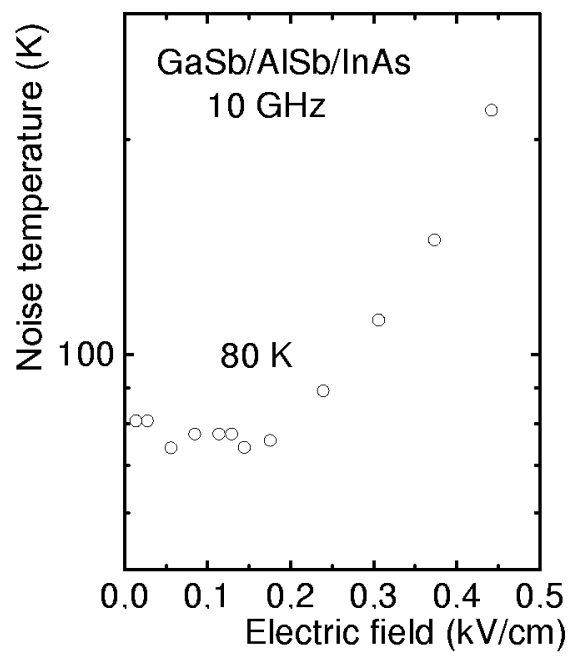

Fig. 3. Field-dependent longitudinal hot-electron noise temperature $T_{\mathrm{n}}$ for $\mathrm{GaSb} / \mathrm{AlSb} / \mathrm{InAs}$ channel and the term $C\left(T_{\mathrm{e}}\right)$ at $300 \mathrm{~K}[3]$.

\subsection{Electron trapping}

The traditional concept of trapping is not applicable to a high-density 2DEG when the Fermi level is located inside the conduction band and the traps in the 


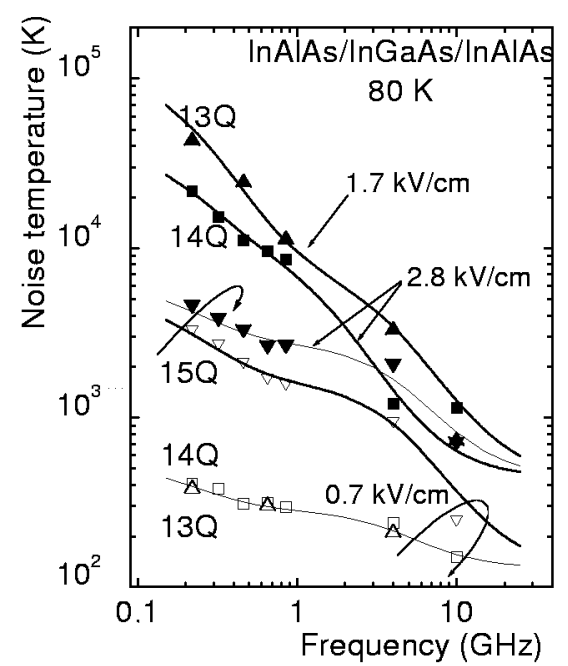

Fig. 4. Frequency-dependent longitudinal noise temperature for InAlAs/InGaAs/ InAlAs 2DEG channels [4]. Solid lines are fitted Lorentzians.

well are completely occupied [4]. Measurements of noise at $80 \mathrm{~K}$ (Fig. 4) yielded two trapping time constants: $\tau_{1}=1 /\left(2 \pi f_{\mathrm{c} 1}\right) \approx 40 \mathrm{ps}$ and $\tau_{2}=1 /\left(2 \pi f_{\mathrm{c} 2}\right) \approx 800 \mathrm{ps}$, where $f_{\mathrm{c} 1}$ and $f_{\mathrm{c} 2}$ are the cutoff frequencies for the corresponding sources of noise. In the channel of $15 \mathrm{Q}$ the electron trapping takes place at equilibrium and at near-equilibrium conditions, but in the channel of $13 \mathrm{Q}$ and $14 \mathrm{Q}$, the electrons must be hot to be trapped efficiently $(E>1 \mathrm{kV} / \mathrm{cm})$. This onset field is lower than that for the real-space transfer, and the electrons cannot surmount the barrier before they are trapped. These and other experiments show that the traps are located in InAlAs. The results of a self-consistent solution of coupled Schrödinger-Poisson equations suggest that the active traps are located at $0.25 \mathrm{eV}$ below the conduction band edge of InAlAs layer.

\subsection{Hot-electron temperature fluctuations and energy relaxation}

Assuming the electron temperature approximation and the energy gain and loss balance,

$$
k_{\mathrm{B}}\left(T_{\mathrm{e}}-T_{0}\right) / \tau_{\varepsilon}=e v_{\mathrm{d}} E,
$$

one can evaluate the phenomenological energy relaxation time $\tau_{\varepsilon}$ in a 2DEG [5]. Here, $e$ is the elementary charge, $T_{\mathrm{e}}$ is the electron temperature, and $v_{\mathrm{d}}$ is the drift velocity. By applying $T_{n \perp}=T_{\mathrm{e}}[8]$ together with the expression (1) for the supercritically doped heterostructure $\mathrm{H} 090$, the estimated value of $\tau_{\varepsilon}=1.9 \mathrm{ps}$ represents the effective energy relaxation time of both high and low mobility channels. The determination of the electron temperature from the longitudinal noise temperature $T_{\mathrm{n} \|}$ is somehow complicated by the presence of the described noise sources. Under 


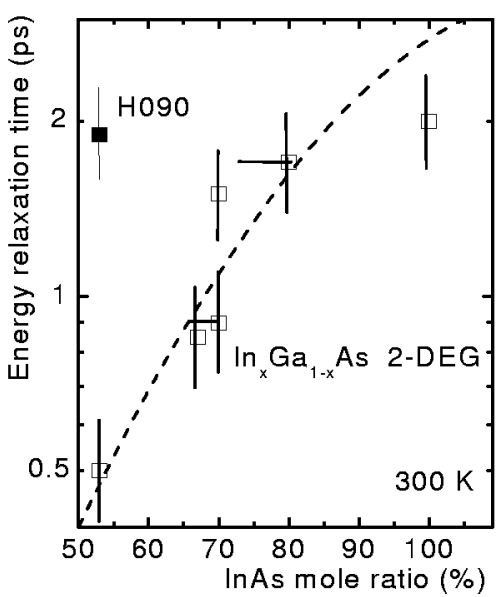

Fig. 5. Hot-electron energy relaxation time in the $\operatorname{In}_{x} \mathrm{Ga}_{1-x}$ As quantum-well channels $[2,3,5]$.

the above mentioned conditions (shift to $\mathrm{X}$ band frequency, variation of dopant density or/and the applied electric field) it is possible to avoid or minimize their influence. However, at moderate electric fields, $T_{\mathrm{n} \|}$ still differs from the electron temperature $T_{\mathrm{e}}$ because of hot-electron temperature fluctuations [9]:

$$
T_{\mathrm{n} \|}=T_{\mathrm{e}}+C\left(T_{\mathrm{e}}\right) .
$$

The extra term $C\left(T_{\mathrm{e}}\right)$ depends on the non-ohmic behavior of the current-voltage characteristic $[10,3,5]$. In the case of small $C\left(T_{\mathrm{e}}\right)$, one can assume that $T_{\mathrm{n} \|} \cong T_{\mathrm{e}}$, and then the expression (1) gives an estimate of $\tau_{\varepsilon}$. Figure 5 presents the obtained values of energy relaxation time at room temperature.

\section{Conclusions}

Noise technique was applied to investigate non-equilibrium fast and ultrafast kinetic processes in heterostructures containing a 2DEG at equilibrium. Sources of noise caused by "thermal" motion of hot electrons, hot-electron temperature fluctuations, real-space-transfer and fast trapping are discussed.

\section{Acknowledgments}

Support under award N00014-01-1-0828 of the Office of Naval Research within NICOP Program and under contract V-019-2001 of the Lithuanian National Science and Education Foundation is gratefully acknowledged. 


\section{References}

[1] H.L. Hartnagel, R. Katilius, A. Matulionis, Microwave Noise in Semiconductor Devices, Wiley, New York 2001.

[2] L. Ardaravičius, J. Liberis, Lith. Phys. J. 40, 357 (2000).

[3] L. Ardaravičius, J. Liberis, A. Matulionis, B.Ya. Mel'tser, V.A. Solov'ev, T.V. Shubina, S.V. Ivanov, P.S. Kop'ev, Mater. Sci. Forum, in press.

[4] A. Matulionis, L. Ardaravičius, J. Liberis, V. Aninkevičius, D. Gasquet, in: Proc. $X V I$ ICNF, Ed. G. Bosman, World Sci., Singapore 2001, p. 517.

[5] A. Matulionis, V. Aninkevičius, J. Liberis, Microelectron. Reliab. 40, 1803 (2000).

[6] G. Tuttle, H. Kromer, J.H. English, J. Appl. Phys. 65, 5239 (1989).

[7] S. Ašmontas, G. Valušis, J. Liberis, L. Subačius, Sov. Phys.-Semicond. 14, 1373 (1990).

[8] R. Katilius, A. Matulionis, Fluct. Noise Lett. 1, R81 (2001).

[9] J.X. Jang, J. Li, C.F. Musante, K.S. Yngveson, Appl. Phys. Lett. 66, 1983 (1995).

[10] Sh.M. Kogan, A.Ya. Shulman, Sov. Phys.-Solid State 9, 1771 (1968). 